\title{
Combined analysis of KamLAND and Borexino neutrino signals from Th and U decays in the Earth's interior
}

\author{
G.L. Fogli, ${ }^{1,2}$ E. Lisi, ${ }^{2}$ A. Palazzo,${ }^{3}$ and A.M. Rotunno ${ }^{1,2}$ \\ ${ }^{1}$ Dipartimento Interateneo di Fisica "Michelangelo Merlin," \\ Via Amendola 173, 70126 Bari, Italy \\ ${ }^{2}$ INFN, Sezione di Bari, \\ Via Orabona 4, 70126, Bari, Italy \\ ${ }^{3}$ AHEP Group, Institut de Física Corpuscular - C.S.I.C./Universitat de Valéncia, \\ Edificio Institutos de Paterna, P.O. Box 22085, E46071 Valencia, Spain
}

\begin{abstract}
The KamLAND and Borexino experiments have detected electron antineutrinos produced in the decay chains of natural thorium and uranium (Th and U geoneutrinos). We analyze the energy spectra of current geoneutrino data in combination with solar and long-baseline reactor neutrino data, with marginalized three-neutrino oscillation parameters. We consider the case with unconstrained Th and U event rates in KamLAND and Borexino, as well as cases with fewer degrees of freedom, as obtained by successively assuming for both experiments a common $\mathrm{Th} / \mathrm{U}$ ratio, a common scaling of $\mathrm{Th}+\mathrm{U}$ event rates, and a chondritic $\mathrm{Th} / \mathrm{U}$ value. In combination, KamLAND and Borexino can reject the null hypothesis (no geoneutrino signal) at $5 \sigma$. Interesting bounds or indications emerge on the $\mathrm{Th}+\mathrm{U}$ geoneutrino rates and on the $\mathrm{Th} / \mathrm{U}$ ratio, in broad agreement with typical Earth model expectations. Conversely, the results disfavor the hypothesis of a georeactor in the Earth's core, if its power exceeds a few TW. The interplay of KamLAND and Borexino geoneutrino data is highlighted.
\end{abstract}

PACS numbers: 14.60.Pq, 91.35.-x, 28.50.Hw, 26.65.+t

\section{CONTEXT}

The study of low-energy electron antineutrinos $\left(\bar{\nu}_{e}\right)$ emitted in the decay chains of uranium (U), thorium (Th), and potassium $(\mathrm{K})$ in the Earth's interior - the so-called geoneutrinos - is raising increasing interest in both particle physics and Earth sciences, as recently reviewed in [1, 2].

From the viewpoint of particle physics, there has been dramatic progress in understanding the flavor evolution and oscillations of neutrinos [3], and in refining their low-energy, low-background detection techniques. In particular, the detection of the global Th $+\mathrm{U}$ geoneutrino flux in the KamLAND [4, 5] and Borexino [6] experiments through the inverse beta decay reaction

$$
\bar{\nu}_{e}+p \rightarrow n+e^{+}\left(E_{\nu}>1.806 \mathrm{MeV}\right),
$$

represents a milestone in this field. ${ }^{1}$ In perspective, measurements at different locations might constrain the relative Th and U abundances in different reservoirs (e.g., crust versus mantle), especially if some directional sensitivity could be achieved [1].

From the viewpoint of Earth sciences, the heat-producing elements U, Th and K, despite their relatively low natural abundances, bear on outstanding and debated issues. Their global amounts should reflect different condensation histories in the primitive solar nebula, which presumably led to partial escape of the moderately "volatile" K and to complete capture of the "refractory" U and Th. Therefore, the mass abundance ratio of Th and U in the Earth ( Th/U hereafter) is expected to be the same as in the most pristine meteorite samples, the so-called carbonaceous chondrites [2],

$$
\frac{\mathrm{Th}}{\mathrm{U}} \simeq 3.9 \text { (chondritic estimate) } .
$$

The radial distribution of $\mathrm{U}$, Th and $\mathrm{K}$ should instead reflect subsequent Earth differentiation processes, as these elements are both "lithophile" (preferring mantle and crust silicates to core metals) and "incompatible" (preferring crust melts to mantle residues). Within the mantle, slow convection processes may have further redistributed the radiogenic elements in several possible ways [2].

\footnotetext{
${ }^{1}$ Geo- $\nu$ from $\mathrm{K}$ decay are below threshold for the reaction (1).
} 
In this field, connecting geophysical quantities to particle physics observables requires some modeling of the U, Th, and $\mathrm{K}$ distributions, on both planetary and local scales. For instance, a correlation is expected between the radiogenic heat production rate $H$ and the geoneutrino event rate $R$ from $\mathrm{Th}+\mathrm{U}$ sources, within large uncertainties induced, e.g., by the unknown distribution of radiogenic elements in the mantle. Various $(H, R)$ correlation plots have been discussed in [1] under rather general and plausible assumptions [including the estimate in (2)] and conservative uncertainties. In particular, the results of [1] for KamLAND (see Figs. 23 and 30 therein) can be approximated as

$$
\frac{H(\mathrm{Th}+\mathrm{U})}{\mathrm{TW}} \simeq(1.11 \pm 0.14) \times \frac{R(\mathrm{Th}+\mathrm{U})}{\mathrm{TNU}}-25.0 \quad(\text { KamLAND, } 6 \mathrm{TW} \lesssim H \lesssim 40 \mathrm{TW}),
$$

where 1 TNU (terrestrial neutrino unit) corresponds to $10^{32}$ events per target proton per year, and the quoted error provides a sort of "maximum allowed range" for the $(H, R)$ correlation band. The above estimate holds for $H \gtrsim 6 \mathrm{TW}$, which provides a total KamLAND signal in excess of the "guaranteed" minimum contribution from the Earth's crust: $R(\mathrm{Th}+\mathrm{U}) \gtrsim 24$ TNU [1]. On the other hand, Eq. (3) is not applicable beyond the "fully radiogenic" limit $H(\mathrm{Th}+\mathrm{U}) \simeq 40 \mathrm{TW}[1]$, which, adding an estimated potassium contribution $H(\mathrm{~K}) \simeq 5 \mathrm{TW}$ [7], would saturate the global Earth's heat flow, $H_{\oplus} \simeq 45$ TW [8, 9].

Positive correlations are also expected among the expected event rates in different experiments, since they probe the same geoneutrino sources, although weighted differently by the inverse square law for the fluxes. In particular, it is rather plausible to assume that KamLAND (KL) and Borexino (BX) probe the same average Th/U ratio, so that

$$
\frac{R(\mathrm{U})_{\mathrm{BX}}}{R(\mathrm{Th})_{\mathrm{BX}}} \simeq \frac{R(\mathrm{U})_{\mathrm{KL}}}{R(\mathrm{Th})_{\mathrm{KL}}}
$$

Even if the primordial proportions of Th and $U$ in the Earth were different from the estimate in (2), the known geochemical similarity of Th and $\mathrm{U}$ in different reservoirs would support the above assumption.

Correlations among absolute rates (rather than ratio of rates) may be more model-dependent. For instance, a comparison of the KamLAND (KL) and Borexino (BX) uranium event rates estimated in [1] for a wide range of admissible Earth models (see Table 12 and Fig. 24 therein) suggests an approximate scaling law,

$$
R(\mathrm{U})_{\mathrm{BX}} \simeq 1.15 R(\mathrm{U})_{\mathrm{KL}},
$$

within a relatively small spread $(<5 \%)$ in the coefficient. A scaling coefficient $>1$ is to be expected, since Borexino probes a thicker crust than KamLAND. Its spread, however, may actually be larger than 5\%, since the local (and sizable) crust contributions at the two sites are not expected to have significant covariances, thus reducing the overall correlation [10]. Nevertheless, within the large uncertainties affecting current geoneutrino event rates, an approximate scaling assumption as in Eq. (5) can still provide a useful guidance in the data analysis, and will be used later.

Summarizing, the most general analysis of the available KamLAND and Borexino data involves four geoneutrino degrees of freedom $\left(N_{D}=4\right)$, namely, the thorium and uranium event rates in the two experiments. This parameter space can be reduced by assuming either of the two Eqs. (4). If the estimate in (2) is also imposed, a single degree of freedom remains $\left(N_{D}=1\right)$. The main purpose of this work is to analyze geoneutrino data, and to discuss their implications, in cases with $N_{D}=4,3,2$, and 1 . A special case is also discussed, where an additional degree of freedom is provided by the unknown power of a hypothetical georeactor in the Earth's core [11].

Our paper is structured as follows. In Section II we discuss our approach to the geoneutrino analysis, with particular attention to the theoretical and experimental energy spectra and their uncertainties. Since low-energy neutrino oscillations - constrained by solar and long-baseline reactor data-affect the extraction of geoneutrino signals, the marginalization of the three-neutrino oscillation parameters in a global fit is also discussed. In Section III we show the results of our analysis in four relevant cases $\left(N_{D}=4,3,2\right.$, and 1), and discuss their implications. In general, we find results in agreement with typical Earth model expectations, although often within large uncertainties. An interesting interplay between KamLAND and Borexino data emerges in all cases with $N_{D} \leq 3$. Finally, we show that the georeactor hypothesis is disfavored by the data. Our conclusions are summarized in Section IV.

\section{METHOD}

In this Section we describe some aspects of our analysis, concerning the geoneutrino and reactor energy spectra, the marginalization of oscillation parameters, the input data from KamLAND and Borexino, and the relevant geoneutrino degrees of freedom under increasingly restrictive assumptions about the relative event rates. 


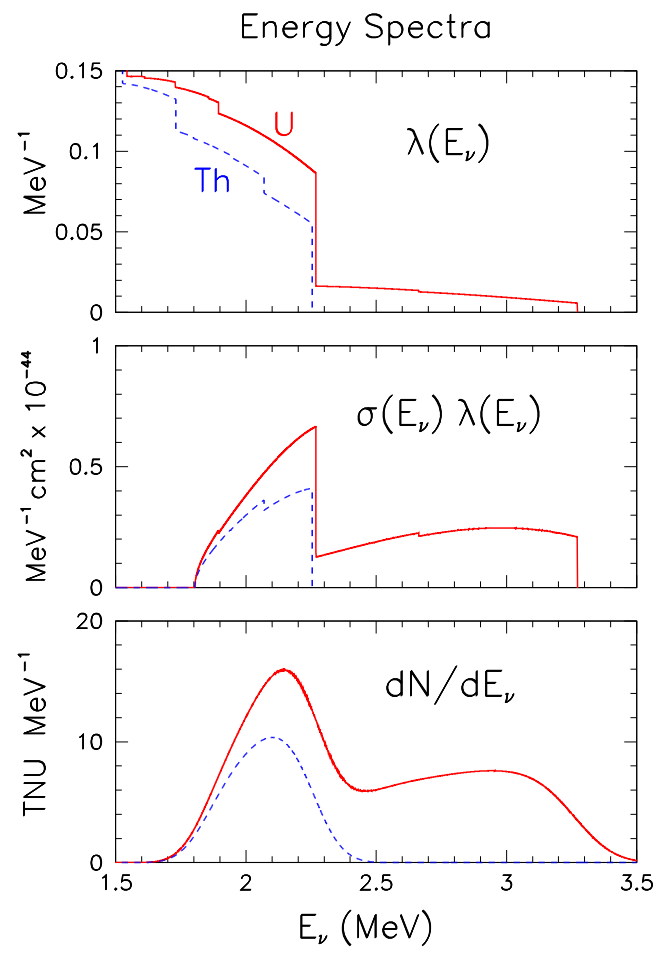

FIG. 1: Geoneutrino spectra computed in this work, as a function of the neutrino energy $E_{\nu}$. Upper panel: spectra from U and Th decay chains (solid and dashed curves, respectively). Middle panel: spectra multiplied by the cross section for inverse beta decay. Lower panel: observable event spectra, including typical energy resolution effects. The results refer to a $\bar{\nu}_{e}$ flux of $10^{6} / \mathrm{cm}^{2} / \mathrm{s}$ (all panels), and to $10^{32}$ target protons (lower panel).

\section{A. Geoneutrino energy spectra}

The reaction (1) allows to detect (a fraction of) the $4 \bar{\nu}_{e}$ and the $6 \bar{\nu}_{e}$ produced in the decay chain of the ${ }^{232} \mathrm{Th}$ and the ${ }^{238} \mathrm{U}$ nuclei, respectively, as described at length in [1], to which we refer the reader for details. We perform our own calculation of the corresponding energy spectra, based on experimental nuclear data from [12] and theoretical inputs from [13].

Our results are reported in Fig. 1. The upper panel shows the computed energy spectra $\lambda(E)$ from the Th and $\mathrm{U}$ decay chains, assuming in both cases a reference $\bar{\nu}_{e}$ flux of $10^{6} / \mathrm{cm}^{2} / \mathrm{s}$. The spectra are normalized to unit area for $E_{\nu} \geq 0$ (lower range not shown). From our spectra we estimate that the flux fraction above the $1.806 \mathrm{MeV}$ threshold is $0.151 / 4(\mathrm{Th})$ and $0.384 / 6(\mathrm{U})$. The middle panel of Fig. 1 shows the spectra times the inverse beta decay cross section [14]. We estimate the average cross sections as $\sigma_{\mathrm{Th}}=12.9 \times 10^{-46} \mathrm{~cm}^{2}$ and $\sigma_{\mathrm{U}}=40.5 \times 10^{-46} \mathrm{~cm}^{2}$, corresponding to the event rates $R(\mathrm{Th})=4.07 \mathrm{TNU}$ and $R(\mathrm{U})=12.8 \mathrm{TNU}$ for a reference $\bar{\nu}_{e}$ flux of $10^{6} / \mathrm{cm}^{2} / \mathrm{s}$. Since $1 \mathrm{~kg}$ of natural Th emits $16.2 \times 10^{6} \bar{\nu}_{e} / \mathrm{s}$ from ${ }^{232} \mathrm{Th}$ decays, and $1 \mathrm{~kg}$ of natural U emits $74.1 \times 10^{6} \bar{\nu}_{e} / \mathrm{s}$ from ${ }^{238} \mathrm{U}$ decays [1], the event rates and the natural mass abundances of Th and $\mathrm{U}$ for a given source are related by

$$
\frac{R(\mathrm{Th})}{R(\mathrm{U})}=6.96 \times 10^{-2} \frac{\mathrm{Th}}{\mathrm{U}} .
$$

The lower panel of Fig. 1 shows the typical effect of finite energy resolution in liquid-scintillator detectors such as KamLAND and Borexino. The event rate spectra appear to be significantly smeared out, in the range $E_{\nu} \simeq 1.7-$ 3.5 MeV. Separation of Th and U contribution requires, in principle, an accurate determination of relative event rates above and below $E_{\nu} \simeq 2.5 \mathrm{MeV}$. Experimentally, one does not measure $E_{\nu}$ but the observable "prompt" energy $E_{p}$ associated to the final-state positron and its annihilation [4 $[\underline{6}]$,

$$
E_{p}=T_{e^{+}}+2 m_{e}=E_{\nu}-\left(m_{n}-m_{p}\right)+m_{e} \simeq E_{\nu}-0.782 \mathrm{MeV} .
$$

Accounting for smearing effects on $E_{p}$, approximate geoneutrino energy windows for $\mathrm{Th}$ and $\mathrm{U}$ events are then $E_{p}(\mathrm{Th}) \in[0.9,1.7] \mathrm{MeV}$ and $E_{p}(\mathrm{U}) \in[0.9,2.6] \mathrm{MeV}$, respectively. 


\section{B. Reactor energy spectra}

Concerning KamLAND, we have described our approach to the reactor spectra and data analysis in previous works [3, 15 17], to which we refer the reader for details. Concerning Borexino, we integrate available information on position, type and average power of European and world reactors from several public sources, see e.g. 18], and adopt the typical power fractions of fuel components as suggested in [6]. Since the oscillated reactor spectra at Borexino are largely averaged over time and over many (and very long) baselines - besides being smeared by energy resolution effects - more accurate information is not really needed for our purposes.

\section{Marginalization of oscillation parameters}

In KamLAND and Borexino, geoneutrino events can be distinguished by reactor and background events only statistically, on the basis of their different energy spectra. The reactor spectra are significantly affected by threeneutrino oscillations governed by the squared mass gap $\delta m^{2}=m_{2}^{2}-m_{1}^{2}$ and by the mixing angles $\theta_{12}$ and $\theta_{13}$ [3]. The pattern of $\delta m^{2}$-driven oscillations clearly emerges in KamLAND reactor spectra after pathlengths $L \sim O\left(10^{2}\right) \mathrm{km}[5]$, while the pattern is largely averaged out in the case of Borexino, where $L \sim O\left(10^{3}\right) \mathrm{km}[6]$. Complete averaging of oscillations can be assumed, to a good approximation, for geoneutrinos [1].

The statistical separation of geoneutrino, reactor and background spectra in KamLAND and Borexino depends thus on the oscillation parameters $\left(\delta m^{2}, \theta_{12}, \theta_{13}\right)$ which, in turn, are also constrained by solar neutrino data [3]. We perform a combined analysis of KamLAND and Borexino data, together with all solar neutrino data, updating our previous work [17, 19]. In particular, we include the latest Gallium experiment event rates [20, 21] and the low-energy threshold data from the Sudbury Neutrino Observatory (SNO) [22]. We also consider both low- and high-metallicity options for the Standard Solar Model [23]. The statistical analysis involves a 7-dimensional manifold, spanned by

$$
\left\{\delta m^{2}, \theta_{12}, \theta_{13} ; R(\mathrm{Th})_{\mathrm{KL}}, R(\mathrm{U})_{\mathrm{KL}}, R(\mathrm{Th})_{\mathrm{BX}}, R(\mathrm{U})_{\mathrm{BX}}\right\}
$$

plus a number of nuisance parameters which account for systematic uncertainties via the pull method [24] (see also the next subsection).

In the global data fit, the marginalization of the four geoneutrino rates $R$ provides bounds on the low-energy oscillation parameters $\left(\delta m^{2}, \theta_{12}, \theta_{13}\right)$, which will be discussed elsewhere. In this work we are interested in the complementary case, where the marginalization of $\left(\delta m^{2}, \theta_{12}, \theta_{13}\right)$ provides constraints on the Th and U event rates in KamLAND and Borexino.

\section{KamLAND and Borexino data}

Experimental geoneutrino spectra are usually presented in terms of number of events $N_{i}$ observed in bins of prompt energy (or a related variable). The total number of events $N$ corresponds to an event rate $R$ at the detector via

$$
N=\varepsilon T R,
$$

where $\varepsilon$ is the detection efficiency, and $T$ is target exposure in units of $10^{32}$ protons $\times$ year, with $R$ expressed in TNU.

We use the latest KamLAND spectral data after an exposure $T=2.44 \times 10^{32}$ protons $\times$ year [5], with small-width bins in the geoneutrino range $E_{p}<2.6 \mathrm{MeV}$ [25]. We include the energy-dependent function $\varepsilon\left(E_{p}\right)$ from [5], which implies average detection efficiencies $\varepsilon_{\mathrm{Th}}=0.69$ and $\varepsilon_{\mathrm{U}}=0.78$ for Th and $\mathrm{U}$ events, respectively 25. A Poisson $\chi^{2}$ function is constructed [15] to account for statistical fluctuations. We introduce five systematic pulls, one for the energy scale uncertainty, and four for the normalization of: ( $i$ ) reactor events; ( $i i)$ all events; (iii) ground-state and (iv) excited-state contributions to the ${ }^{13} \mathrm{C}(\alpha, n)^{16} \mathrm{O}$ background.

Figure 2 shows the experimental KamLAND spectrum in terms of prompt energy, as well as the partial and total contributions to the theoretical spectrum (background, reactor signal, and geoneutrino signal) at the best fit in the parameter space (8). The geoneutrino signal is more pronounced at low energies, consistently with a relatively large contribution from Th decay $\left(E_{p} \lesssim 1.7 \mathrm{MeV}\right)$. Our geoneutrino fit results are very similar to the official ones [5]; moreover, we reproduce the fit of [25] in terms of total $\mathrm{Th}+\mathrm{U}$ rate versus $\mathrm{Th}-\mathrm{U}$ rate asymmetry (not shown). We find that the null hypothesis (no geoneutrino signal in KamLAND) is rejected at $2.9 \sigma$.

Concerning Borexino, we use the spectral data for an effective exposure $\varepsilon T=0.152 \times 10^{32}$ protons $\times$ year, and in terms of the light yield $Y$ for positron events - approximately equal to $Y \simeq 500 \times E_{p} / \mathrm{MeV}[6]$. The data from the americium-beryllium source calibration in [6] allow us to improve this approximation for $Y$, as well as to infer the energy resolution width in Borexino. Our Poisson $\chi^{2}$ statistics includes two systematic pulls for the normalization of 


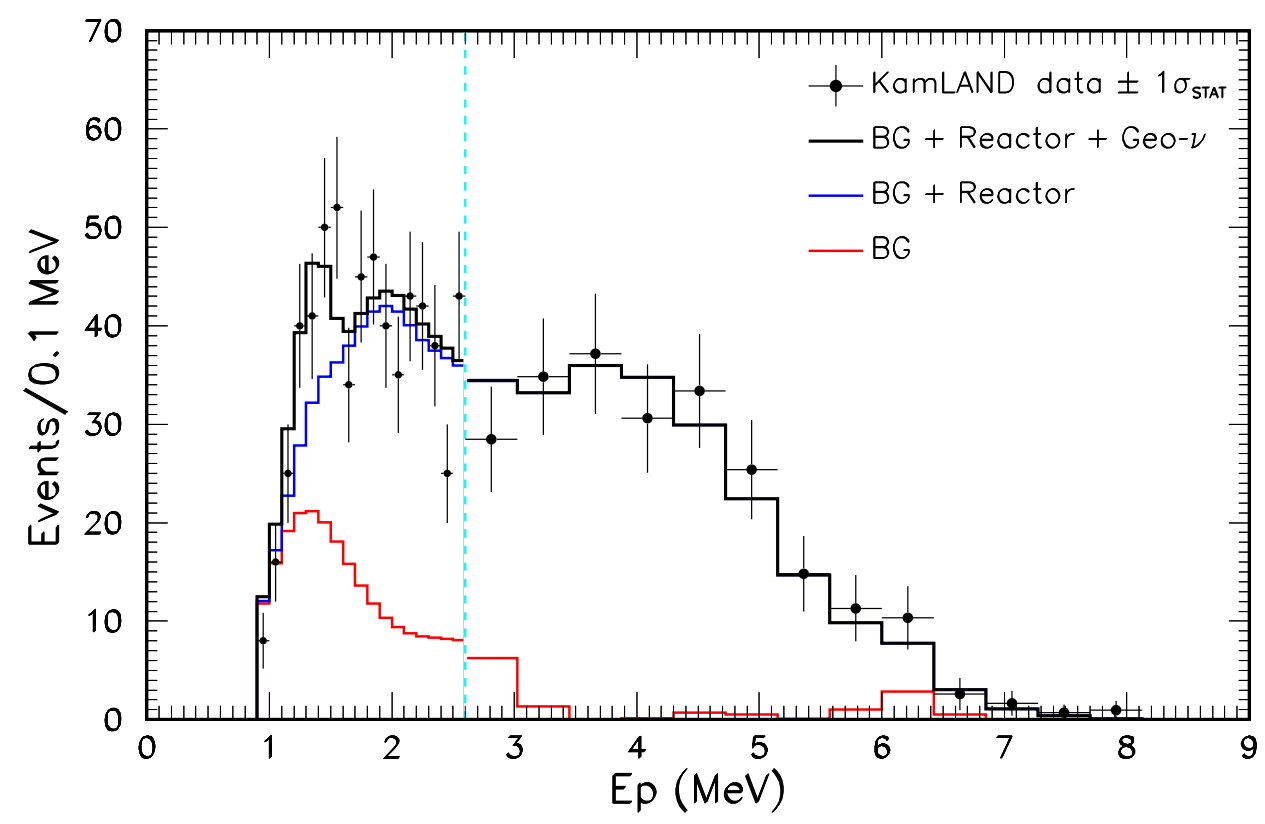

FIG. 2: KamLAND event spectrum as a function of the observed prompt energy $E_{p}$. Data points and backround (BG) estimates are taken from [5, 25]; note the narrower binning in the geoneutrino energy range $E_{p}<2.6 \mathrm{MeV}$ [25]. The histogram represents our best-fit spectrum, with cumulative contributions from background, plus reactor, plus geoneutrino events.

all events and of reactor-only events. Since the background is very small in Borexino, its systematic uncertainties are negligibile for our purposes.

Figure 3 shows the experimental Borexino spectrum in terms of light yield, as well as the separate contributions to our best-fit spectrum, in analogy with Fig. 2. Note the clear geoneutrino signal, which covers the whole expected range $E_{p} \in[0.9,2.6] \mathrm{MeV}(Y \in[450,1300])$, consistent with a leading contribution from U decay. Also in this case, we are able to reproduce quite well the official geoneutrino fit results and plots of [6] (not shown). We find that the null hypothesis in Borexino is rejected at $4.1 \sigma$.

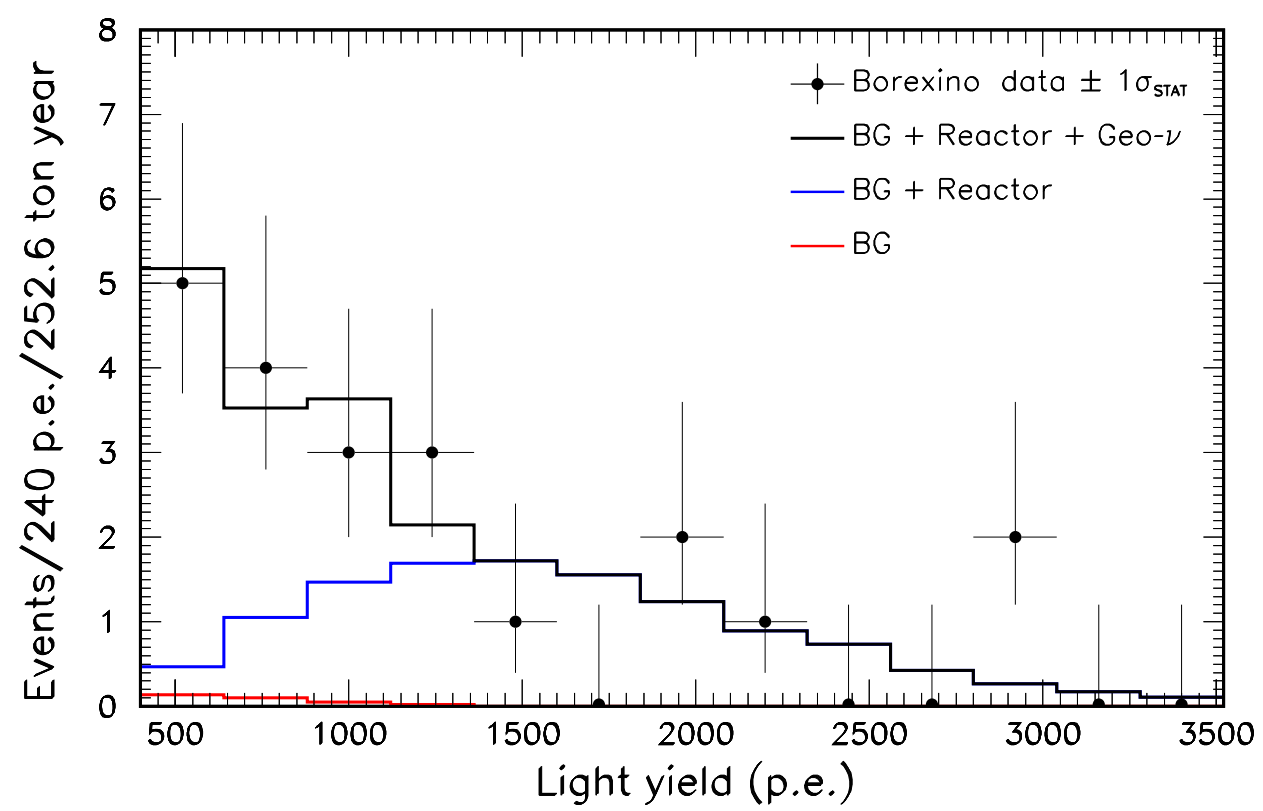

FIG. 3: Borexino event spectrum as a function of the light yield for positron events (p.e.). Data points and background (BG) estimates are taken from [6]. The histogram represents our best-fit spectrum, with cumulative contributions from background, plus reactor, plus geoneutrino events. 
TABLE I: Summary of adopted degrees of freedom and constraints.

\begin{tabular}{clccc}
\hline \hline$N_{D}$ & Constraints & $R(\mathrm{Th}+\mathrm{U})_{\mathrm{KL}}$ & $(\mathrm{Th} / \mathrm{U})_{\mathrm{KL}}$ & $R(\mathrm{Th}+\mathrm{U})_{\mathrm{BX}}(\mathrm{Th} / \mathrm{U})_{\mathrm{BX}}$ \\
\hline 4 & None & free & free & free \\
3 & $(\mathrm{Th} / \mathrm{U})_{\mathrm{BX}}=(\mathrm{Th} / \mathrm{U})_{\mathrm{KL}}$ & free & free & free \\
2 & $(\mathrm{Th} / \mathrm{U})_{\mathrm{BX}}=(\mathrm{Th} / \mathrm{U})_{\mathrm{KL}}$ and $R_{\mathrm{BX}}=1.15 R_{\mathrm{KL}}$ & free & free & - \\
1 & $(\mathrm{Th} / \mathrm{U})_{\mathrm{BX}}=(\mathrm{Th} / \mathrm{U})_{\mathrm{KL}}=3.9$ and $R_{\mathrm{BX}}=1.15 R_{\mathrm{KL}}$ & free & - & - \\
\hline \hline
\end{tabular}

\section{E. Analyses with 4, 3, 2, and 1 degrees of freedom}

After marginalization of the oscillation parameters in (8), the most general geoneutrino parameter space is spanned by four event rates. We find it useful to define four equivalent geoneutrino degrees of freedom $\left(N_{D}=4\right)$, namely, the total rate and the Th/U ratio probed by KamLAND and Borexino,

$$
\begin{aligned}
R(\mathrm{Th}+\mathrm{U})_{\mathrm{KL}} & =R(\mathrm{Th})_{\mathrm{KL}}+R(\mathrm{U})_{\mathrm{KL}} \\
(\mathrm{Th} / \mathrm{U})_{\mathrm{KL}} & =\left[R(\mathrm{Th})_{\mathrm{KL}} / R(\mathrm{U})_{\mathrm{KL}}\right] / 6.96 \times 10^{-2} \\
R(\mathrm{Th}+\mathrm{U})_{\mathrm{BX}} & =R(\mathrm{Th})_{\mathrm{BX}}+R(\mathrm{U})_{\mathrm{BX}} \\
(\mathrm{Th} / \mathrm{U})_{\mathrm{BX}} & =\left[R(\mathrm{Th})_{\mathrm{BX}} / R(\mathrm{U})_{\mathrm{BX}}\right] / 6.96 \times 10^{-2}
\end{aligned}
$$

One can reduce the degrees of freedom to $N_{D}=3$ by assuming that KL and BX probe essentially the same Th/U ratio,

$$
(\mathrm{Th} / \mathrm{U})_{\mathrm{BX}}=(\mathrm{Th} / \mathrm{U})_{\mathrm{KL}}
$$

If, in addition, the scaling law in Eq. (5) is assumed, then $N_{D}=2$. Finally, if the chondritic Th/U estimate in (2) is also assumed, then $N_{D}=1$. These four options, involving an increasing Earth model dependence for decreasing $N_{D}$, are summarized in Table 1 .

A final remark is in order. As discussed in the next Section, the allowed ranges for the KamLAND and Borexino geoneutrino degrees of freedom may extend beyond plausible expectations, where the constraints in Eqs. (2), (5) and (14) are not really justified by any Earth model. Therefore, while the analysis for $N_{D}=4$ is completely general, the results of constrained analyses $\left(N_{D} \leq 3\right)$ must be taken with a grain of salt.

\section{F. Analysis with an additional degree of freedom: the georeactor}

It has been proposed [11] that there could be enough uranium in the Earth's core to naturally start a nuclear fission chain over geological timescales, with a typical power (at the current epoch) of $P_{\text {geo }} \simeq 3-10$ TW [26]. This hypothesis is disfavored by various geochemical and geophysical arguments [28]. Particle physics offers an independent probe of the hypothesis, since a georeactor would alter the observable energy (and time) spectra of $\bar{\nu}_{e}$ events [27]. In particular, we reported in [16] an analysis of earlier KamLAND data in the energy and time domain, providing an upper bound $P_{\text {geo }} \lesssim 13$ TW at $95 \%$ C.L. Further KamLAND and Borexino data have reduced the upper bound to $\lesssim 6.2$ TW at $90 \%$ C.L. [5] and $\lesssim 3 \mathrm{TW}$ at $95 \%$ C.L. [6], respectively.

Here we update our previous analysis [16], by assuming a contribution from a georeactor at the center of the Earth (with unconstrained $P_{\text {geo }}$ ) in the KamLAND and Borexino energy spectra, for each of the four cases in Table 1 . With respect to [16], the current analysis does not include the event time information, which has not been released by the experiments [5], [6].

\section{RESULTS}

In this Section we describe the results of our analysis, in terms of both joint and separate bounds on the Th/U and $R(\mathrm{Th}+\mathrm{U})$ variables. 

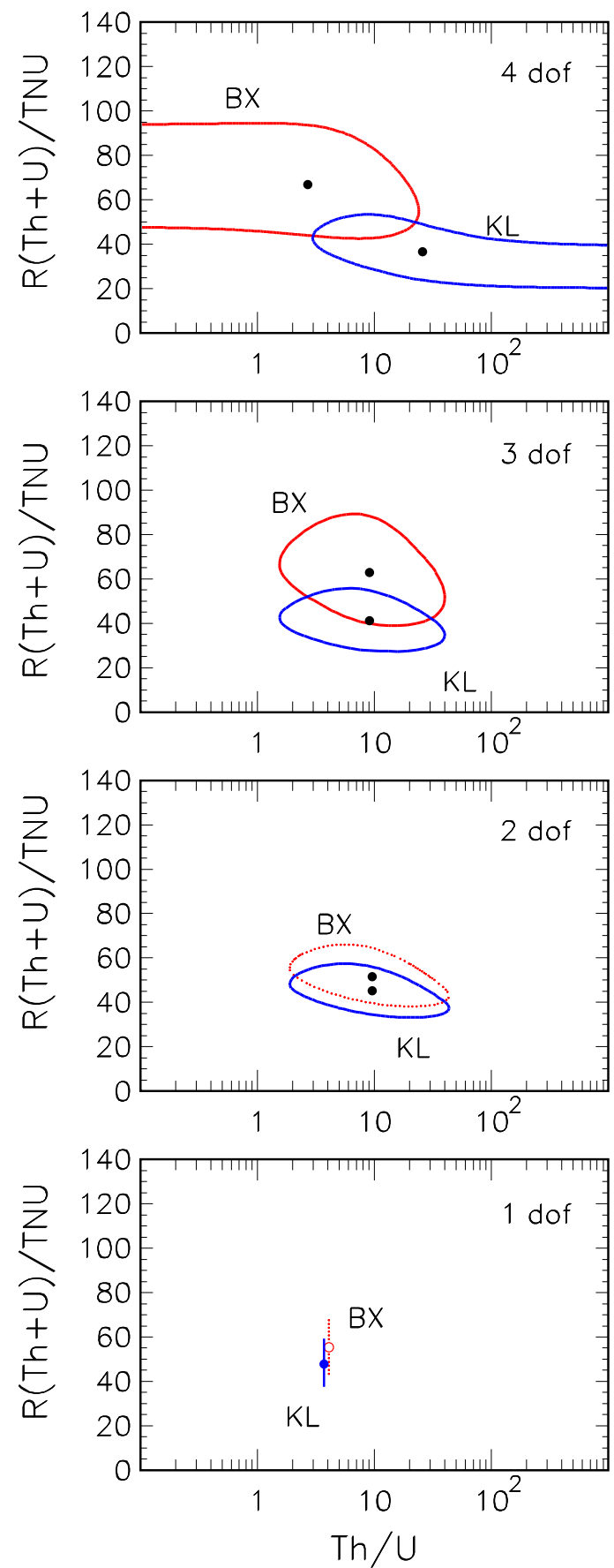

FIG. 4: KamLAND (KL) and Borexino (BX) geoneutrino analysis in the plane charted by the total rate $R(\mathrm{Th}+\mathrm{U})$ and by the mass abundance ratio $\mathrm{Th} / \mathrm{U}$. The curves represents $1 \sigma$ contours $\left(\Delta \chi^{2}=1\right)$ around the best-fit points (thick dots). From top to bottom, the degrees of freedom decrease from $N_{D}=4$ to $N_{D}=1$, as reported in Table 1

\section{A. Joint $1 \sigma$ regions for $\mathrm{Th} / \mathrm{U}$ and $\boldsymbol{R}(\mathrm{Th}+\mathrm{U})$}

Figure 4 shows the $1 \sigma$ contours $\left(\Delta \chi^{2}=1\right)$ in the plane charted by the total event rate $R(\mathrm{Th}+\mathrm{U})$ and by the mass abundance ratio Th/U for KamLAND and Borexino. The degrees of freedom decrease from $N_{D}=4$ to $N_{D}=1$ from top to bottom, according to the constraints in Table I.

The upper panel $\left(N_{D}=4\right)$ shows that both KamLAND and Borexino place upper and lower bounds to the total event rate $R(\mathrm{Th}+\mathrm{U})$. These bounds are consistent with typical Earth model expectations, which span the $1 \sigma$ range 29-41 TNU for KamLAND and 34-48 TNU for Borexino (see Table 11 in [1]). However, neither KamLAND nor 
TABLE II: Best fits and $1 \sigma$ ranges from the data analysis with degrees of freedom $N_{D} \leq 4$. Event rates $R$ are expressed in TNU. Derived or fixed numbers are given in brackets.

\begin{tabular}{ccccc}
\hline \hline$N_{D}$ & $R(\mathrm{Th}+\mathrm{U})_{\mathrm{KL}}$ & $(\mathrm{Th} / \mathrm{U})_{\mathrm{KL}}$ & $R(\mathrm{Th}+\mathrm{U})_{\mathrm{BX}}$ & $(\mathrm{Th} / \mathrm{U})_{\mathrm{BX}}$ \\
\hline 4 & $36.8_{-16.1}^{+16.2}$ & $25.9_{-22.9}^{+\infty}$ & $66.9_{-23.8}^{+27.3}$ & $2.7_{-2.7}^{+20.2}$ \\
3 & $41.3_{-12.6}^{+14.0}$ & $9.1_{-7.4}^{+23.5}$ & $63.0_{-24.0}^{+26.0}$ & {$\left[9.1_{-7.4}^{+23.5}\right]$} \\
2 & $45.1_{-11.2}^{+11.8}$ & $9.6_{-7.6}^{+33.7}$ & {$\left[51.7_{-12.9}^{+13.6}\right]$} & {$\left[9.6_{-7.6}^{+33.7}\right]$} \\
1 & $47.7_{-11.2}^{+11.2}$ & {$[3.9]$} & {$\left[54.9_{-12.9}^{+12.9}\right]$} & {$[3.9]$} \\
\hline \hline
\end{tabular}

Borexino can currently determine Th and U separately. In particular, KamLAND is compatible with all events being from Th decay $(\mathrm{Th} / \mathrm{U}=\infty)$, while Borexino is compatible with all events being from $\mathrm{U}$ decay $(\mathrm{Th} / \mathrm{U}=0$ ), as anticipated in the context of Figs. 2 and 3, respectively. However, a broad range of Th/U values appears to be compatible with both KamLAND and Borexino results at $1 \sigma$; this range excludes the extreme cases with null Th or $\mathrm{U}$ signal, and includes the chondritic value $\mathrm{Th} / \mathrm{U}=3.9$. Therefore, it makes sense to reduce the degrees of freedom by imposing that the two experiments probe the same $\mathrm{Th} / \mathrm{U}$ ratio as in Eq. (14).

The second panel $\left(N_{D}=3\right)$ shows the results of such exercise, providing both upper and lower $1 \sigma$ limits on the $\mathrm{Th} / \mathrm{U}$ ratio, with a best fit which is only a factor of $\sim 2$ higher than the chondritic value. The total rate estimates are not significantly altered with respect to the case with $N_{D}=4$. Therefore, under the rather general assumption in Eq. (14), the combination of KamLAND and Borexino data starts to be sensitive to the global Th/U ratio of the Earth, although only at the $\sim 1 \sigma$ level; as discussed below, current Th/U constraints disappear at $\sim 1.5 \sigma$.

The results in the third panel $\left(N_{D}=2\right)$ include, in addition, the approximate scaling assumption in Eq. (5). In this case, the KamLAND parameters $R(\mathrm{Th}+\mathrm{U})$ and $\mathrm{Th} / \mathrm{U}$ are conventionally taken as free, while the corresponding Borexino parameters are derived (hence the "dotted" BX contour in the panel). In this case, the best fits for the total rates are located slightly above the quoted Earth model expectations (29-41 TNU for KamLAND and 34-48 TNU for Borexino [1] ), with $1 \sigma$ uncertainties at the level of $\sim 30 \%$, dominated by KamLAND data. Concerning Th/U, the best fits and $1 \sigma$ ranges are not significantly altered with respect to the previous case with $N_{D}=3$.

The comparison of the three panels with $N_{D}=4,3$ and 2 shows that the current constraints on the total rates and on the Th/U ratio are approximately independent: they do not significantly affect each other within present data. The weak negative correlation in the $1 \sigma$ contours reflects the fact the overall rate $R(\mathrm{Th}+\mathrm{U})$ increases somewhat faster for larger $\mathrm{U}$ contribution as compared to Th contribution, the latter being confined at low energy (see the spectra in Fig. 1).

Finally, the results in the lower panel of Fig. $4\left(N_{D}=1\right)$ include, in addition to the previous constraints, the chondritic estimate in (2).$^{2}$ In this case, the estimated KL total rate is $R(\mathrm{Th}+\mathrm{U})_{\mathrm{KL}}=47.7 \pm 11.2 \mathrm{TNU}$, the BX total rate being a factor $\sim 1.15$ higher by construction, $R(\mathrm{Th}+\mathrm{U})_{\mathrm{BX}}=54.9 \pm 12.9 \mathrm{TNU}$. These results show a preference for Earth models with relatively high expectations in Th and U contents, although within very large uncertainties at present. We report in Table II a numerical summary of the $1 \sigma$ ranges for the total rate and Th/U ratio, in each of the four cases considered.

In the most constrained case $\left(N_{D}=1\right)$, where the total rate error is reduced to $\sim 23 \%$, it makes sense to infer indications about the associated radiogenic heat $H(\mathrm{Th}+\mathrm{U})$ via the approximate $(H, R)$ correlation in Eq. (3). By fixing the $\mathrm{KL}$ rate at its central value, $R(\mathrm{Th}+\mathrm{U})_{\mathrm{KL}}=47.7 \mathrm{TNU}$, one would obtain an allowed range $H(\mathrm{Th}+\mathrm{U}) \simeq 21-$ $35 \mathrm{TW}$, somewhat above the plausible expectations of 14-18 TW (see Fig. 23 in [1]). However, including the $1 \sigma$ rate uncertainties, the allowed range in significantly enlarged: $H(\mathrm{Th}+\mathrm{U}) \simeq 10-49 \mathrm{TW}$. The upper value is not particularly meaningful, being larger than the "fully radiogenic" limit of $\sim 40 \mathrm{TW}$. The lower value of $\sim 10 \mathrm{TW}$, however, exceeds the "guaranteed" contribution from Th and U in the crust ( 6 TW [1]), and suggests, indirectly, the presence of an additional contribution from a different reservoir - which can be naturally identified with the mantle.

In conclusion, the combination of KamLAND and Borexino data brings to surface some intriguing - although still statistically weak - pieces of information: $(i)$ preferred Th/U values in broad agreement with chondritic expectations; (ii) slight preference for relatively high Th and U contents in the Earth; and (iii) hints of a mantle contribution to the total geoneutrino signal. We remark that these indications emerge only at the $\sim 1 \sigma$ level from the current, low-statistics data samples.

\footnotetext{
${ }^{2}$ In the panel, the Th/U coordinates of KL and BX are slightly displaced from 3.9 for the sake of clarity.
} 

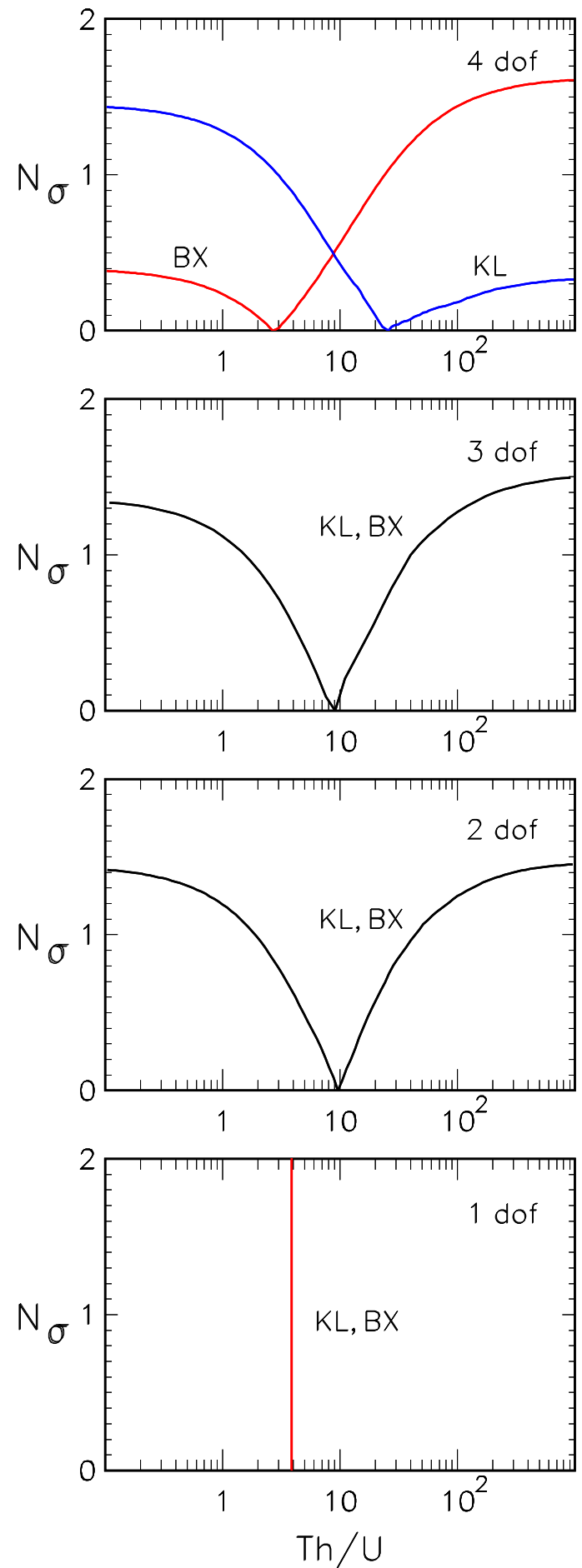

FIG. 5: KamLAND and Borexino analysis: constraints on the Th/U ratio in terms of standard deviations, $N_{\sigma}=\sqrt{\Delta \chi^{2}}$.

\section{B. Separate bounds on $\mathrm{Th} / \mathrm{U}$ and $R(\mathrm{Th}+\mathrm{U})$}

In this section we discuss the separate projections of the previous results onto the variables $\mathrm{Th} / \mathrm{U}$ and $R(\mathrm{Th}+\mathrm{U})$, in terms of standard deviations from their best fit $\left(N_{\sigma}=\sqrt{\Delta \chi^{2}}\right)$.

Figure 5 shows the constraints on the Th/U ratio from KamLAND and Borexino. In the upper panel $\left(N_{D}=4\right)$, one can see at a glance that KamLAND and Borexino data place lower and upper $1 \sigma$ limits on Th/U, respectively, but have no significant sensitivity in the opposite directions. The bounds are still statistically weak, as they vanish 

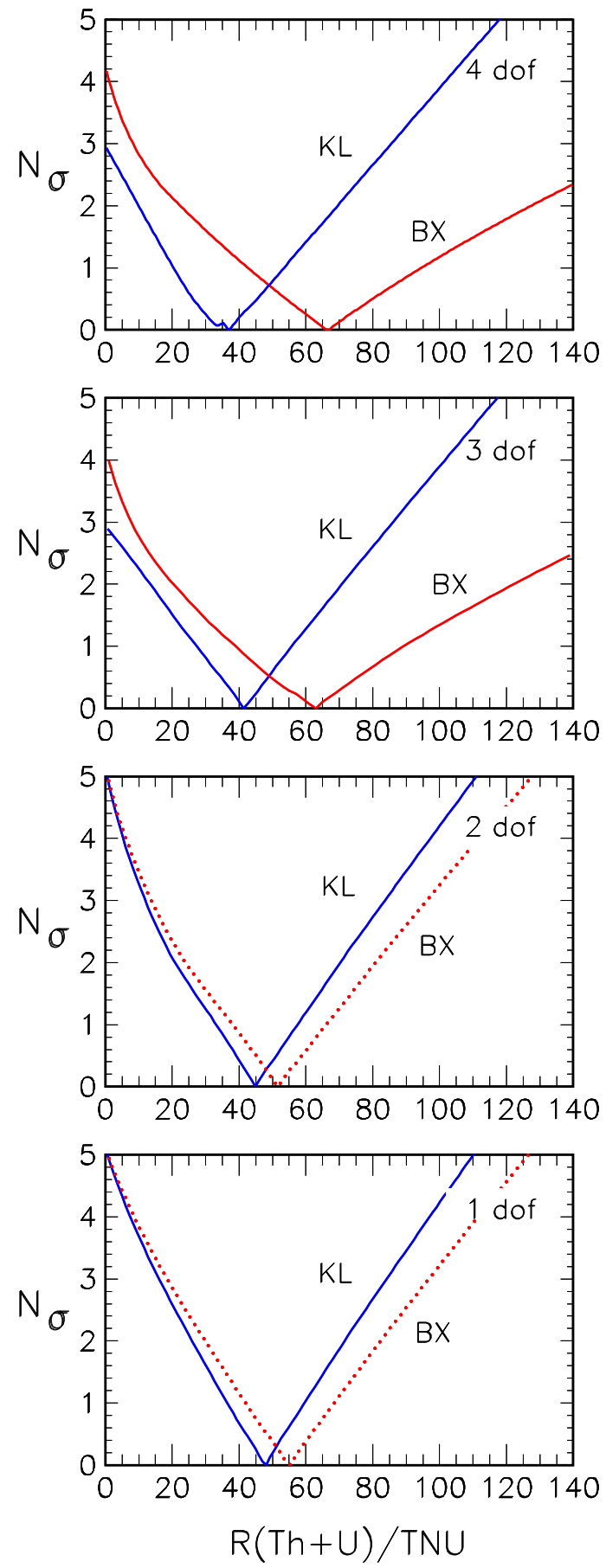

FIG. 6: KamLAND and Borexino analysis: constraints on the total rate $R(\mathrm{Th}+\mathrm{U})$ in terms of standard deviations $N_{\sigma}$.

at $\sim 1.3 \sigma$ in KamLAND and at $\sim 1.6 \sigma$ in Borexino. The middle panels show that KamLAND and Borexino provide joint limits on $\mathrm{Th} / \mathrm{U}$ at the $1 \sigma$ level, with no significant variation between the cases with $N_{D}=3$ and $N_{D}=2$. The lower panel is just a "Dirac delta" at $\mathrm{Th} / \mathrm{U}=3.9$.

Figure 6 shows the constraints on the total event rate $R(\mathrm{Th}+\mathrm{U})$ in KamLAND and Borexino. The two upper panels shows that the null hypothesis of no geoneutrino signal is rejected at $2.9 \sigma$ in KamLAND and at $4.1 \sigma$ in Borexino, almost independently of the Th/U constraint in Eq. (14). The two lower panels show the significant error reduction induced by the scaling law assumption in Eq. (5), which strengthens the null hypothesis rejection at the $5 \sigma$ level. By comparing the two lower panels, it appears that the chondritic assumption in (2) has a minor impact on combined rate constraints. 
In conclusion, current indications on the $\mathrm{Th} / \mathrm{U}$ ratio emerge only at the level of $\sim 1 \sigma$, while global geoneutrino signals emerge at $\gtrsim 3 \sigma$, reaching $\sim 5 \sigma$ in constrained combinations. Bounds on $\mathrm{Th}+\mathrm{U}$ rates and on the Th/U ratio are largely uncorrelated. Detailed $1 \sigma$ ranges for both free and derived parameters are reported in Table I.

\section{Bounds on the power of a hypothetical georeactor}

We have repeated the various analyses by including a spectral contribution from a hypothetical georeactor with unknown power $P_{\text {geo }}$, located at the center of the Earth. The results disfavor this hypothesis in all cases, if $P_{\text {geo }}$ exceeds a few TW. In the most general case (unconstrained geoneutrino and georeactor event rates), we find that KamLAND and Borexino place the $2 \sigma$ upper limits $P_{\text {geo }} \lesssim 4.1 \mathrm{TW}$ and $\lesssim 6.7 \mathrm{TW}$, respectively. In combination, the joint KamLAND+Borexino limit reads

$$
P_{\text {geo }} \lesssim 3.9 \mathrm{TW} \text { at } 2 \sigma(\lesssim 5.2 \mathrm{TW} \text { at } 3 \sigma)
$$

almost independently of the chosen geoneutrino degrees of freedom. This is to be expected, since the georeactor spectrum extends well above the geoneutrino energy range.

Our combined constraints on $P_{\text {geo }}$ appear to be dominated by Borexino data, but do not improve upon the official Borexino limit (quoted as $P_{\text {geo }} \lesssim 3 \mathrm{TW}$ at $2 \sigma[6]$ ), presumably because we cannot include information in the time domain, which is currently unpublished. Our analysis of older KamLAND data [16] has indeed shown that the time spectra can add significant constraints to $P_{\text {geo }}$.

\section{CONCLUSIONS}

We have performed a detailed analysis of current geoneutrino events from Th and U decay chains as detected in KamLAND [5] and Borexino [6], within the more general context of low-energy neutrino oscillation data from long-baseline reactor and solar sources, and of a broad range of Earth model expectations taken from [1].

The relevant parameter space is spanned by the total $\mathrm{Th}+\mathrm{U}$ event rates and the $\mathrm{Th} / \mathrm{U}$ ratio in the two experiments, while the oscillation parameters $\left(\delta m^{2}, \theta_{12}, \theta_{13}\right)$ are marginalized away. Constrained analyses with fewer degrees of freedom are obtained by successively assuming for both experiments a common Th/U ratio [Eq. (14)], a common scaling of Th $+\mathrm{U}$ event rates [Eq. (5)], and a fixed (chondritic) Th/U value [Eq. (2)]. Cases with a hypothetical georeactor, involving an additional degree of freedom, are also considered.

The results are in agreement with typical Earth model expectations, although within still large uncertainties. The global $\mathrm{Th}+\mathrm{U}$ geoneutrino signal emerges at $2.9 \sigma$ and $4.1 \sigma$ in KamLAND and Borexino, respectively, and can reach the overall $5 \sigma$ level in combination. The data disfavor the hypothesis of a georeactor, and limit its power to $P_{\text {geo }} \lesssim 3.9 \mathrm{TW}$ at $2 \sigma\left(\right.$ or $P_{\text {geo }} \lesssim 5.2 \mathrm{TW}$ at $\left.3 \sigma\right)$.

Weaker - but potentially interesting — pieces of information emerge at the $\sim 1 \sigma$ level, including: $(i)$ preferred Th/U values in broad agreement with chondritic expectations; ( $i i)$ slight preference for relatively high Th and U contents in the Earth; and ( ii i ) possible hints of a (mantle) contribution in excess of the "guaranteed" signal from the crust. Significantly higher statistics, possibly from new large-volume detectors and with some directional sensitivity, will be needed to promote these intriguing indications to more robust signals improving our understanding of the Earth's interior.

\section{Acknowledgments}

E.L. thanks G. Fiorentini, A. Ianni and W.F. McDonough for many useful discussions about geoneutrino physics in the last few years. The work of G.L.F., E.L. and A.M.R. is partly supported by the Italian Ministero dell'Istruzione, dell'Università e della Ricerca (MIUR) through the Progetto di Rilevante Interesse Nazionale (PRIN) "Fisica Astroparticellare: Neutrino ed Universo Primordiale," and partly by the Istituto Nazionale di Fisica Nucleare (INFN) through the research initiative "Fisica Astroparticellare FA51." The work of A.P. is supported by the Spanish Ministerio de Educación y Ciencia (MEC) under the I3P program, by the Spanish Grants FPA2008-00319, CSD2009-00064, PROMETEO/2009/091, and by the European network UNILHC (PITN-GA-2009-237920). 
[1] G. Fiorentini, M. Lissia and F. Mantovani, "Geo-neutrinos and Earth's interior," Phys. Rept. 453, 117 (2007).

[2] W.F. McDonough and R. Arevalo Jr., "Uncertainties in the composition of Earth, its core and silicate sphere," Proceedings on Neutrino 2008, XXIII International Conference on Neutrino Physics and Astrophysics (Christchurch, New Zealand, 2008), ed. by J. Adams, F. Halzen, and S. Parke, Journal of Physics Conference Series 136, 022006 (2008).

[3] G. L. Fogli, E. Lisi, A. Marrone and A. Palazzo, "Global analysis of three-flavor neutrino masses and mixings," Prog. Part. Nucl. Phys. 57, 742 (2006).

[4] KamLAND Collaboration, T. Araki et al., "Experimental investigation of geologically produced antineutrinos with KamLAND," Nature 436, 499 (2005).

[5] KamLAND Collaboration, S. Abe et al., "Precision Measurement of Neutrino Oscillation Parameters with KamLAND," Phys. Rev. Lett. 100, 221803 (2008).

[6] Borexino Collaboration, G. Bellini et al., "Observation of Geo-Neutrinos," Phys. Lett. B 687, 299 (2010).

[7] R. Arevalo Jr, W.F. McDonough, and M. Luong, "The K/U ratio of the silicate Earth: Insights into mantle composition, structure and thermal evolution," Earth Planet. Sci. Lett. 278, 361 (2009).

[8] H.N. Pollack, S.J. Hurter, and J.R. Johnson, "Heat Flow from the Earth's Interior: Analysis of the Global Data Set," Rev. Geophys. 31, 267-280 (1993).

[9] C. Jaupart, S. Labrosse, and J.-C. Mareschal, "Temperatures, Heat and Energy in the Mantle of the Earth," in Treatise on Geophysics, Vol. 7, pp. 253-303, edited by G. Schubert (Elsevier, Oxford, 2007).

[10] G.L. Fogli, E. Lisi, A. Palazzo, and A.M. Rotunno, "Geoneutrinos: A systematic approach to uncertainties and correlations," Proceedings of Neutrino Geophysics (Honolulu, Hawaii, 2005), edited by S.T. Dye, Earth, Moon, and Planets 99, 111 (2007).

[11] J.M. Herndon, "Sub-structure of the inner core of the Earth," Proc. Natl. Acad. Sci. U.S.A. 93(2), 646 (1996); "Nuclear georeactor origin of oceanic basalt ${ }^{3} \mathrm{He} /{ }^{4} \mathrm{He}$, evidence, and implications," ibidem 100(6), 3047 (2003).

[12] Isotopes Project database, available at ie.lbl.gov.

[13] H. Behrens and J. Janecke, "Numerical tables for beta decay and electron capture," (Springer Verlag, Berlin, 1969).

[14] A. Strumia and F. Vissani, "Precise quasielastic neutrino nucleon cross section," Phys. Lett. B 564, 42 (2003).

[15] G.L. Fogli, E. Lisi, A. Marrone, D. Montanino, A. Palazzo and A.M. Rotunno, "Solar neutrino oscillation parameters after first KamLAND results," Phys. Rev. D 67, 073002 (2003).

[16] G.L. Fogli, E. Lisi, A. Palazzo and A.M. Rotunno, "KamLAND neutrino spectra in energy and time: Indications for reactor power variations and constraints on the georeactor," Phys. Lett. B 623, 80 (2005).

[17] G.L. Fogli, E. Lisi, A. Marrone, A. Melchiorri, A. Palazzo, A.M. Rotunno, P. Serra, J. Silk, and A. Slosar, "Observables sensitive to absolute neutrino masses. II," Phys. Rev. D 78, 033010 (2008).

[18] Databases of nuclear power plants are available at: International Nuclear Safety Center (www.insc.anl.gov); Global Resource Information Database (GRID)—Europe (www.grid.unep.ch); International Atomic Energy Agency (www .iaea . org); World Nuclear Association (www.world-nuclear.org).

[19] G.L. Fogli, E. Lisi, A. Marrone, A. Palazzo and A.M. Rotunno, "Hints of $\theta_{13}>0$ from global neutrino data analysis," Phys. Rev. Lett. 101, 141801 (2008).

[20] SAGE Collaboration, J.N. Abdurashitov et al., "Measurement of the solar neutrino capture rate with gallium metal. III: Results for the 2002-2007 data-taking period," Phys. Rev. C 80, 015807 (2009).

[21] F. Kaether, W. Hampel, G. Heusser, J. Kiko and T. Kirsten, "Reanalysis of the GALLEX solar neutrino flux and source experiments," Phys. Lett. B 685, 47 (2010).

[22] SNO Collaboration, B. Aharmim et al., "Low Energy Threshold Analysis of the Phase I and Phase II Data Sets of the Sudbury Neutrino Observatory," arXiv:0910.2984 [nucl-ex].

[23] A. Serenelli, S. Basu, J.W. Ferguson and M. Asplund, "New Solar Composition: The Problem With Solar Models Revisited," arXiv:0909.2668 [astro-ph.SR].

[24] G.L. Fogli, E. Lisi, A. Marrone, D. Montanino and A. Palazzo, "Getting the most from the statistical analysis of solar neutrino oscillations," Phys. Rev. D 66, 053010 (2002).

[25] S. Enomoto, "KamLAND Geoneutrino Studies," talk at the Workshop Neutrino Geoscience 2008 (Sudbury, Ontario, Canada, 2008); available at geonu.snolab.ca.

[26] J.M. Herndon and D.A. Edgerley, arXiv:hep-ph/0501216

[27] R.S. Raghavan, "Detecting a nuclear fission reactor at the center of the earth," arXiv:hep-ex/0208038

[28] W.F. McDonough, "Compositional Models for the Earth's Core," in Treatise on Geochemistry, Vol. II, edited by R.W. Carlson (Elsevier-Pergamon, Oxford, 2003). 